\title{
Supplementary file
}
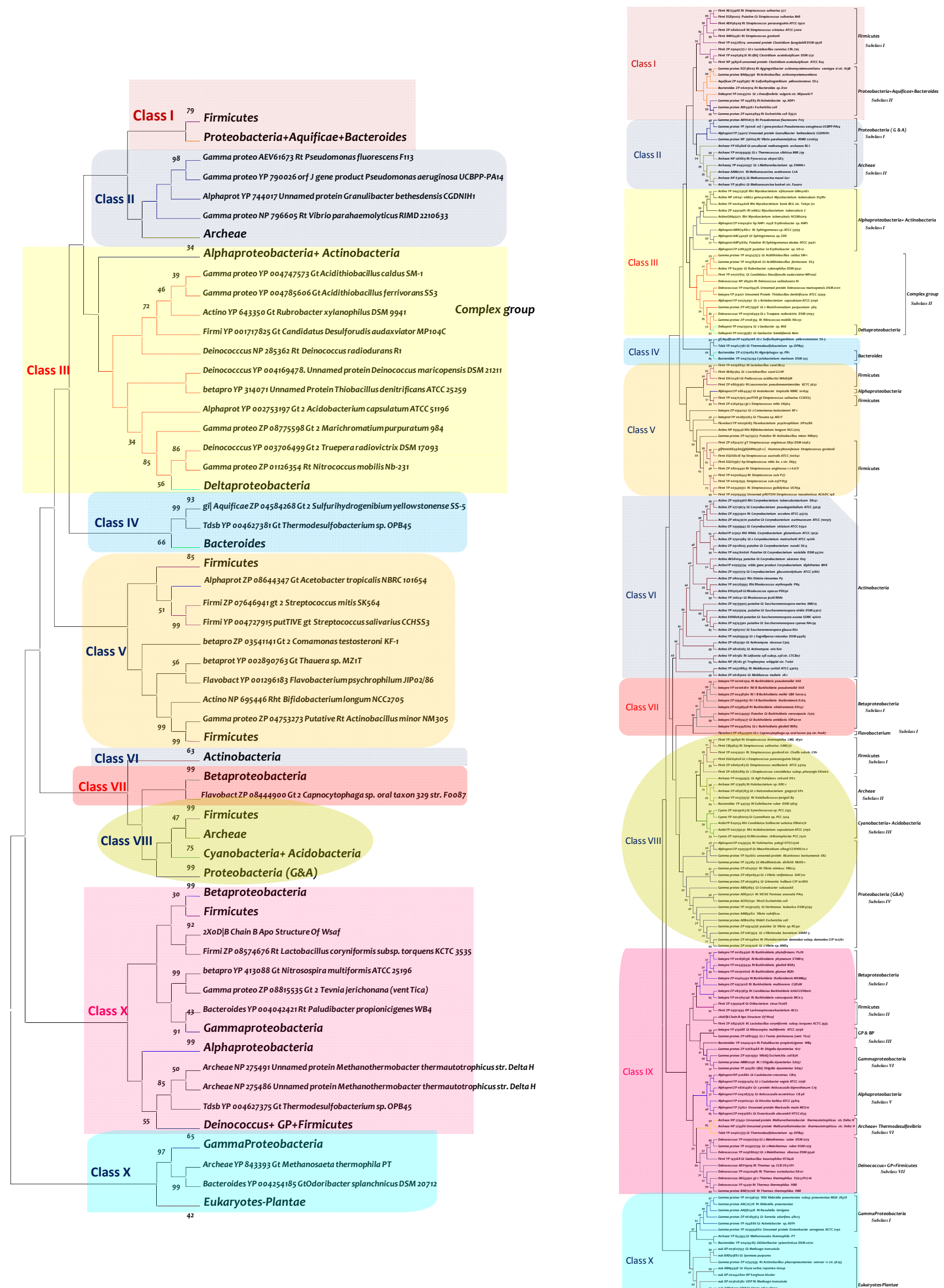
Fig. 1 (supplementary file). A comprehensive phylogenetic tree of Rhamnosyl transferases: The evolutionary history was inferred using the Neighbor-Joining method (Saitou and Nei 1987). The bootstrap consensus tree inferred from 1000 replicates is taken to represent the evolutionary history of the taxa analyzed. The analysis involved 204 amino acid sequences. Only bootstrap value higher than 50 are shown. Evolutionary analyses were conducted in MEGA5. The Bacterial groups taken for study were Proteobacteria (Alpha, Beta, Gamma, Detla), Firmicutes, Actinobacteria, Acidobacteria, Cyanobacteria, Archeae Bacteroides and Deinococcus. Plant specific enzymes were included based on the existence. 